Authors:

Amnon Amir ${ }^{1 *}$

Eitan Ozel ${ }^{2}$

Yael Haberman ${ }^{3}$

Noam Shental $* 2$

* Corresponding authors

1. Microbiome center, Sheba Medical Center, Israel

2. Dept. of Computer Science, The Open University of Israel, Israel

3. Pediatric Gastroenterology, Hepatology and Nutrition Unit, Sheba Medical Center, Israel 


\title{
Title: Achieving pan-microbiome biological insights via the dbBact knowledge base
}

\begin{abstract}
16S rRNA amplicon sequencing provides a relatively cheap culture-independent method for studying the microbial world. Thousands of studies have examined microbial populations in various habitats, yet a global pan-microbiome perspective integrating these studies is still missing. Here we introduce dbBact, an open wiki-like bacterial knowledge base that combines information from hundreds of studies across diverse habitats, creating a collaborative central repository amenable to subsequent analysis. 16S rRNA amplicon sequence variants (ASVs) are manually extracted from each study and assigned multiple ontology-based terms. dbBact currently contains about 850 studies, covering more than 1,300,000 associations between 315,000 ASVs and 3,500 ontology terms. We demonstrate how dbBact can lead to better understanding of underlying bacterial communities and the formulation of novel biological hypotheses. dbBact can be accessed using a website (http://dbbact.org), plugins for qiime2 and Calour, and programmatically, using a REST-API.
\end{abstract}

\section{Introduction}

Bacteria play an important role in the Earth's ecosystem, having a total biomass higher than that of all vertebrates and fish, second only to plants (1). The introduction of 16S rRNA amplicon sequencing as a means for molecular identification has enabled a culture-independent view of such ecosystems (2). Combined with massively parallel sequencing technologies and DNA barcodes (3), 16S rRNA sequencing provides relatively cheap and accurate microbial profiling. This, in turn, has led to a huge surge in the number of $16 \mathrm{~S}$ rRNA studies examining microbial populations in habitats ranging across oceans (4), soil (5), plants (6), animals (7), and large cross-sectional human studies (8-10).

A severe limitation when combining insights from multiple microbiome studies is the complexity of the underlying bacterial populations, ranging from tens of different bacteria in a single saliva sample (11), to thousands in a single soil sample (12). In addition, although the total number of different bacteria is large (e.g., 300000 unique 16S rRNA sequences of length 90bp appear in the Earth Microbiome Project (12)), the number of taxonomic names for describing these bacteria is 
much smaller (about 3500-4000 unique genera and 20000 unique species names appear in NCBITAX (13) and in the Encyclopedia of Life (14)). Moreover, grouping bacteria in higher taxonomic levels may not always maintain the basic habitat properties of many bacteria (12). Therefore, reaching cross-study biological insights should preferably be based on 16S rRNA amplicon sequences rather than on taxonomy.

Recently, amplicon sequence variants (ASVs) derived using denoising methods such as Deblur (15), DADA2 (16) and UNOISE2 (17) have been introduced as an alternative to OTU picking for identifying bacteria in a given sample. Such denoising methods provide an objective identification of each bacterial sequence in the sample (i.e., independent of external databases or additional bacteria/samples in the experiment), as well as high sequence resolution (a single nucleotide difference in the sequenced region is identified as a different ASV). Therefore, ASVs may serve as cross-study identifiers for bacteria, i.e., a bacterium in different studies will result in the same ASV, even when the studies are processed separately and denoised using different methods (15, 18).

In this paper, we introduce dbBact, a knowledge base for reaching cross-experiment biological insights. dbBact is based on manually collecting genotype-phenotype associations between ASVs and relevant conditions. For clarity, "reserved" dbBact words appear in italics: surveyed studies are referred to as experiments, stored ASVs are sequences, phenotypes are ontology-based terms, and genotype-phenotype associations are called annotations.

Currently, dbBact contains more than 850 studies in various habitats, covering more than 1,300,000 associations between 315,000 ASVs and 3,500 ontology terms. For retrieval, dbBact provides two query types: a single sequence/FASTA file query, asking what is known about these sequences (Figure 1), and a query contrasting two FASTA files, searching for dbBact terms significantly enriched in either of the groups, analogously to gene ontology enrichment analysis $(19,20)$ (Figure 2). By examining the ontology terms associated with each sequence, users can gain insights regarding the biology associated with ASVs of interest.

dbBact differs from other microbial databases in several aspects: (a) Manual annotation: dbBact phenotype-genotype associations are extracted using manual analysis, in contrast to microbial data repositories, such as SRA/EBI, Qiita (21), HMP DACC (10, 22), MGnify (23), FoodmicrobioNet (24), and redBIOM (25), which provide raw experimental data and metadata. In dbBact, the human expert understands the experimental setting and identifies abundant bacteria in different study 
groups, detects contaminants, etc., and these associations are uploaded. (b) Unlimited scope: dbBact accepts studies across all habitats, unlike databases that are highly limited in scope, e.g., human-disease-focused databases (MicroPhenoDB (26), gutMDisorder (27), Disbiome database (28), Peryton (29), and BugSigDB (30) ), or other context-specific databases (database of the healthy mouse microbiome (31) or the sponge microbiome project (32)). (c) Volume and potential growth: to date, the number of studies in dbBact is $\sim 40 \%$ higher than in Qiita, and the number of ASVs is comparable to those in the Earth Microbiome Project. New studies are continually added by the dbBact team. Additionally, as a wiki-like database, we encourage the microbiome community to contribute to dbBact. (d) Structured genotype/phenotype search: observations are uploaded at the ASV level, allowing queries of specific sequences and subsequences. In addition, as phenotypes are designated by terms derived from multiple ontologies, subsequent querying allows for "cross-sectioning" of the data. For example, sequences associated with Crohn's disease and ulcerative colitis both originate from the DOID ontology (33), and will be recalled when querying their "parent" term, "inflammatory bowel disease." (e) Harmonizing studies performed using different variable regions: as uploaded studies may be sequenced using different 16S rRNA variable regions, stored sequences are "linked" through the SILVA database of full-length 16S rRNA genes (34), facilitating cross-region queries. For example, when submitting a query sequence from V1-V2, dbBact seeks the matching full-length 16S rRNA genes in SILVA, then extracts their V4 region, and subsequently retrieves relevant annotations. (f) Data analysis: dbBact provides a set of statistical tools for analyzing new studies and for generating novel biological hypotheses.

Apart from accessing dbBact through its website (http://dbbact.org), it is possible to integrate dbBact in microbiome analysis pipelines through the Qiime2 (35) and Calour (36) plugins, and programmatic query submission is available through the dbBact REST-API interface. A weekly snapshot of the complete dbBact SQL database is available for download, and can be used for local analysis. 


\section{Populating dbBact (wiki-like)}

\section{$\underline{\text { Steps }}$}

\section{a. Biological observation from an amplicon experiment:}

b. Upload related sequences:<smiles>C1C2CC3CC1CC(C2)C3</smiles>

\section{Example}

Certain bacteria are more abundant in children with Crohn's disease compared to healthy adult controls From ljaz et al.

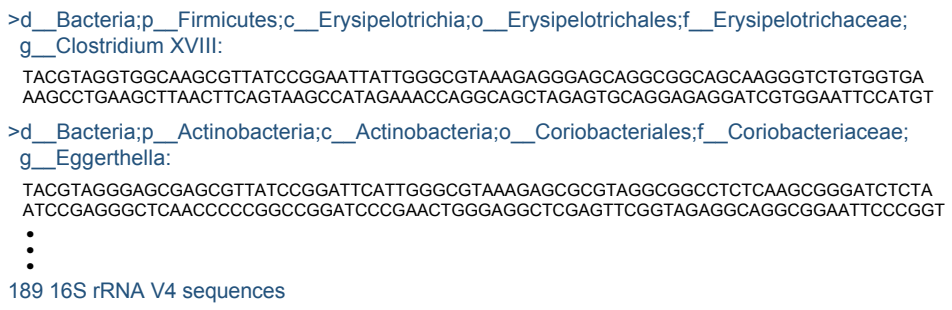
AAGCCTGAAGCTTAACTTCAGTAAGCCATAGAAACCAGGCAGCTAGAGTGCAGGAGAGGATCGTGGAATTCCATGT

$>d \_$_Bacteria; $\ldots$ _Actinobacteria;c_Actinobacteria;o_Coriobacteriales;f_Coriobacteriaceae;

g_Eggerthella:

TACGTAGGGAGCGAGCGTTATCCGGATTCATTGGGCGTAAAGAGCGCGTAGGCGGCCTCTCAAGCGGGATCTCTA ATCCGAGGGCTCAACCCCCGGCCGGATCCCGAACTGGGAGGCTCGAGTTCGGTAGAGGCAGGCGGAATTCCCGGT $\vdots$

189 16S rRNA V4 sequences

\section{c. Enter annotation terms} describing these sequences:

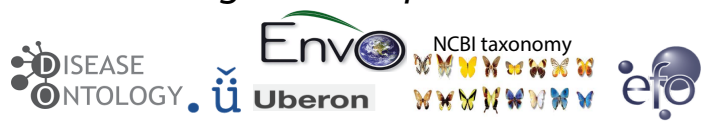

ANNOTATION TYPE: DIFFENTIAL

SOURCE: feces, homo sapiens, glasgow HIGHER IN: crohn's disease, child, obsolete juvenile stage LOWER IN: control, adult

\section{d. Update dbBact via:}
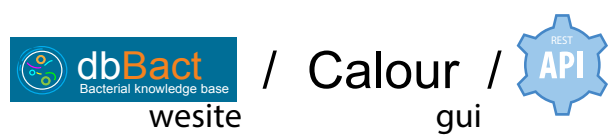

Figure 1: Adding entries to dbBact. Users add new entries in a wiki-like way, by uploading study results. a. For example, analyzing data from Ijaz et al. (37), we identified 189 ASVs that are more abundant in fecal samples of Scottish children with Crohn's disease compared to healthy controls (see Methods section). b. These ASVs are uploaded as a FASTA file. c. Associations between ASVs and phenotypes are called annotations, which are created by assigning a set of ontology terms and predicates that characterize the context. The 189 sequences were annotated as "DIFFERENTIAL," i.e., more abundant in children with Crohn's disease ("HIGHER IN" terms), compared to healthy controls ("LOWER IN" terms). The general background terms common to both groups, i.e., "homo sapiens," "feces" and "glasgow" are designated by "SOURCE." Terms may be selected from several ontologies (e.g., DOID (33), ENVO (38, 39), GAZ (40), UBERON (41), EFO (42), and NCBI Taxonomy (13)), allowing easy and precise annotations. d. Uploading annotations may be performed either through the dbBact website, dedicated clients (i.e., Calour (36)) or by REST-API. For clarity, the following nomenclature holds throughout the manuscript where "reserved" words appear in italics (e.g., experiment, sequence, annotation, term), predicates appear in all caps (e.g., "HIGHER IN," "LOWER IN," “SOURCE”), and specific term names follow the ontology convention of being lower case (e.g., "homo sapiens"). 
a. A single FASTA sequence query

$$
\begin{aligned}
& \text { submit a sequence } \\
& \text { or a single FASTA file }
\end{aligned}
$$

\section{$>$ Clostridium XIVa sequence}

TACGTAGGGGGCAAGCGTTATCCGGATTTACTGGGT

GTAAAGGGAGCGTAGACAGCGTAGACGGCGAAGCA

FASTA AGTCTGAAGTGAAAACCCAGGGCTCAACCCTGGGAC

TGCTITGGAAACTGTITTGCTAGAGTGTCGGAGAGGT AAGTGGAATTCCTA

\section{a1. retrieved annotations}

a2. dbBact word cloud

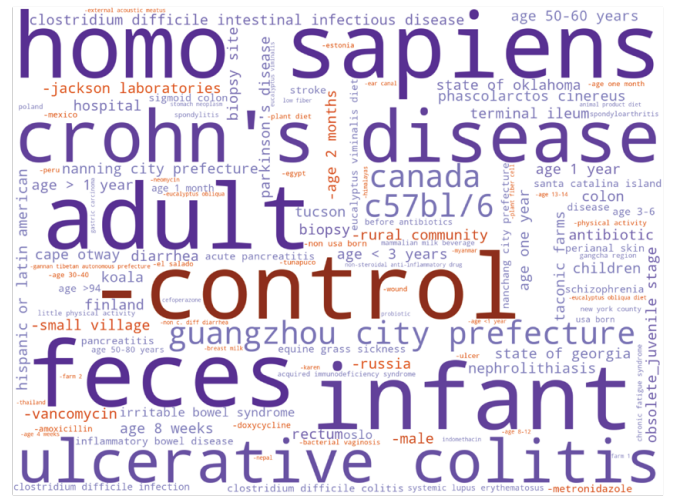

- HIGHER IN: ulcerative colitis; LOWER IN: control; SOURCE: feces, homo sapiens, adult, state of California, united states of America

- HIGHER IN: irritable bowel syndrome; LOWER IN: control; SOURCE: feces, homo sapiens, adult, kingdom of Spain

- HIGHER IN: inflammatory bowel disease, crohns disease, ulcerative colitis, child; LOWER IN: control; SOURCE: feces, homo sapiens, commonwealth of Pennsylvania, united states of America

- HIGHER IN: systemic Iupus erythematosus; LOWER IN: control; SOURCE: feces, homo sapiens, adult, commonwealth of Virginia, united states of America

- HIGHER IN: infant, age 1 year; LOWER IN: adult age 30-40; SOURCE: feces, homo sapiens, Oslo, kingdom of Norway

- HIGHER IN: probiotic; LOWER IN: control; SOURCE: feces, mouse, mus musculus, antibiotics, research facility, Israel

\section{b. A two-group enrichment query}

\section{submit two FASTA files}

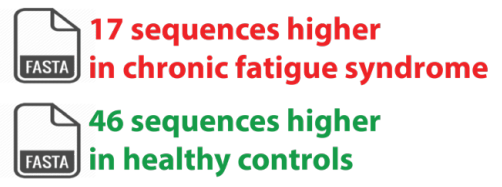

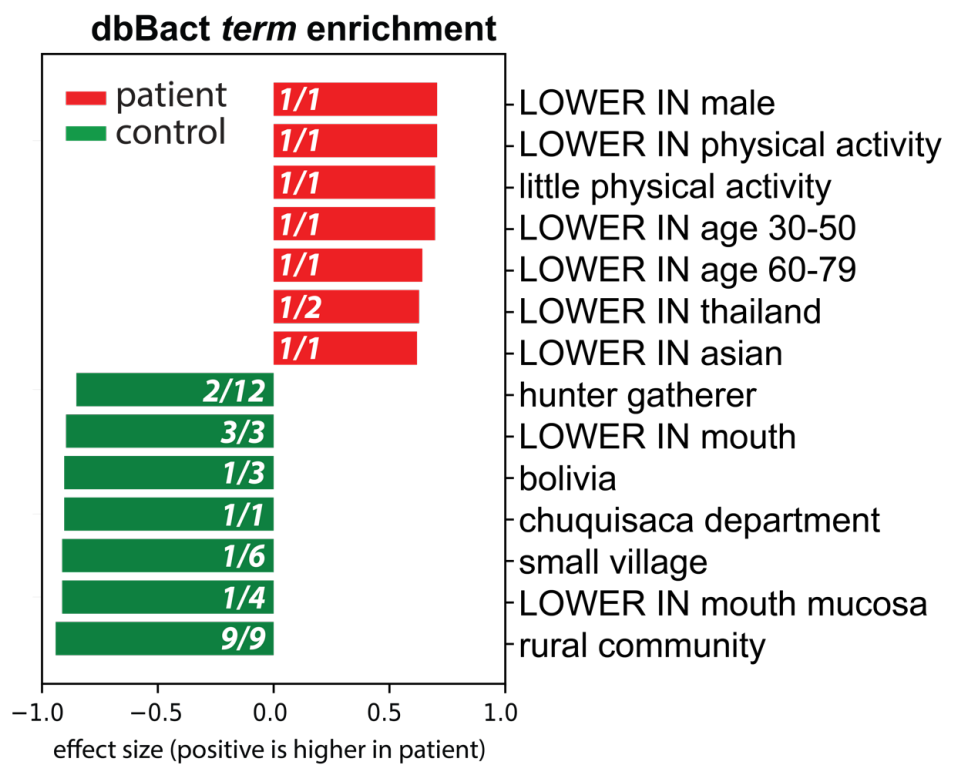

dbBact term enrichment

Figure 2: Two basic query types: a. Uploading a FASTA file of sequences results in a list of the most relevant annotations containing these sequences, and a "word cloud" of best matching terms. In this example, a V4 sequence of Clostridium XIVa, which is highly abundant in fecal samples of chronic fatigue syndrome patients (CSF) (Giloteaux et al., 2016), was submitted. Panel a1 provides representative annotations containing the query sequence (the full list of $\sim 150$ annotations appears in Supplementary File 3). dbBact found 
this sequence to be higher in the disease group than in healthy controls in several studies (ulcerative colitis, irritable bowel disease, and lupus), and in antibiotic-treated mice supplemented with probiotics (last annotation arising from (43)). Panel a2 displays the word cloud summarizing the terms associated with the query sequence, where size corresponds to a term's F-score, while color designates the associated predicate (blue for "SOURCE"/"HIGHER IN" terms, and red color preceded by a minus sign corresponds to "LOWER IN" terms). Hence, this Clostridium XIVa query sequence is associated with human feces in dysbiosis states of “crohn's disease," "ulcerative colitis," "diarrhea," and "c. difficile infection" (a full list of F-scores per term appears in Supplementary File S7). b. When uploading two FASTA files, dbBact identifies enriched terms characterizing each group. For example, 17 and 46 sequences were submitted, corresponding to differentially abundant sequences higher in chronic fatigue syndrome (CFS) patients and in healthy controls, respectively. Bar lengths show the normalized rank-mean difference for the top significantly enriched terms in either bacterial group. Term enrichment is based on a nonparametric rank mean test with FDR $<0.1$ using dsFDR (see term enrichment analysis section in Methods). The numbers in the bar of each term correspond to the number of dbBact experiments in which the term is significantly different between the two sequence groups (numerator), and the total of dbBact experiments containing the term (denominator). Sequences that were more abundant in the CFS group are enriched in terms related to low physical activity, while healthy controls are enriched in terms related to rural and less industrialized communities.

\section{Results}

dbBact: Scope and comprehensiveness

For the sake of reproducibility, we used dbBact release 2021.05 for all analyses presented in the Results section. This release contains approximately 260,000 unique bacterial amplicon sequences, an amount that is on par with the 300,000 sequences observed by the Earth Microbiome Project (12). Sequences arise from over 780 unique experiments, i.e., studies from which observations were added (Figure 3a). Over 5500 dbBact annotations associate these sequences with various phenotypes using ontology derived terms. As each annotation typically includes many sequences, this results in over 960,000 unique genotype-phenotype associations. 


\section{General statistics of dbBact}

The $\sim 780$ dbBact experiments cover a wide range of habitats (Figure S1a), geographic regions (Figure S1b), plant and animal hosts (Figure S1c), human body sites (Figure S1d), and human diseases (Figure 3b). For example, 149 experiments cover diseases, of which 86 are of an anatomical entity (e.g., Crohn's disease or ulcerative colitis), and seven more are defined as metabolic diseases. The most abundant dbBact terms are "united states of america," "homo sapiens," and "feces," each appearing in over 1000 annotations arising from more than 150 different experiments. Most of the other terms appear in less than twenty experiments (Figure 3c). The most prevalent bacterial sequence is E. coli, appearing in over 900 annotations from over 300 experiments (Figure 3d). Although this could reflect the universality of E. coli in various habitats, it may also be due to potential contaminations (44), a reason that may also explain the high prevalence of Staphylococcus (appearing in over $200 \mathrm{dbBact}$ experiments). The number of experiments per sequence follows a power law distribution, with a majority of sequences appearing in a single experiment, yet over 80,000 sequences were observed in more than one experiment and 7000 sequences appeared in at least ten experiments (Figure $3 \mathrm{~d}$ ).

dbBact allows the upload of sequences from several commonly used regions (V1-V2, V3-V4 or V4; see Table S2 for a list of primers). Upon upload, sequences from different regions are "linked" through their full-length $16 \mathrm{~S}$ rRNA sequence in the SILVA database (34) (see Inter-region querying section in Methods). When submitting query sequences from one region, dbBact retrieves all annotations containing the corresponding sequences across all regions (including, naturally, the region from which the query was provided). To demonstrate the usefulness of such "linking," Figure S2 provides several examples of V1-V2 and V3-V4 sequence queries that are successfully characterized based solely on "linked" V4 sequences.

\section{Comprehensiveness of dbBact}

Intra-dbBact estimates. To estimate the comprehensiveness of dbBact, we tested how many bacterial sequences typical of a specific environment (e.g., human feces) have annotations arising from more than one experiment. We selected sequences having an annotation of type "COMMON" (i.e., present in more than half of the samples in an experiment) for each of several terms, and measured the fraction of these sequences that have annotations from another dbBact experiment (Figure 3e). For example, there are 97 experiments having a "COMMON IN homo 
sapiens feces" annotation. Iterating over each of these annotations, about $98 \%$ of the associated sequences appear in more than one experiment. Hence, fecal bacteria are already well covered by dbBact. A similar level of "coverage" occurs for several other human-related terms and for dogs, where almost all sequences were observed in more than a single experiment. Regarding the terms "cow," "soil," "root," and "leaf," about 80\% of the sequences appear in more than a single experiment, whereas the "coverage" of "green turtle" is much lower, indicating that additional experiments are required to capture its full bacterial diversity.

Out-of-sample comprehensiveness. As another example of comprehensiveness, dbBact was tested in a source tracking task, i.e., identifying the host or niche of a sample based on its bacterial composition. Hägglund et al. collected samples from either sewage influent or from freshwater, as well as feces sampled from several animals (rabbit, cat, wild boar, dog, cow and deer), aiming to find unique bacterial footprints of each source (45). We used all sequences present in more than $1 / 3$ of the samples from each group as queries to dbBact resulting in word clouds describing each sample group (Figure 3f). In almost all cases, the notable terms in each word cloud were indicative of the sources of the samples, e.g., sus scrofa for the wild boar fecal sample, allowing accurate source tracking. The only exception was cat fecal samples, which were detected as a combination of cat, dog, and human, probably because of the small number of cat fecal samples present in the current dbBact release. 
bioRxiv preprint doi: https://doi.org/10.1101/2022.02.27.482174; this version posted February 28, 2022. The copyright holder for this preprint (which was not certified by peer review) is the author/funder. All rights reserved. No reuse allowed without permission.

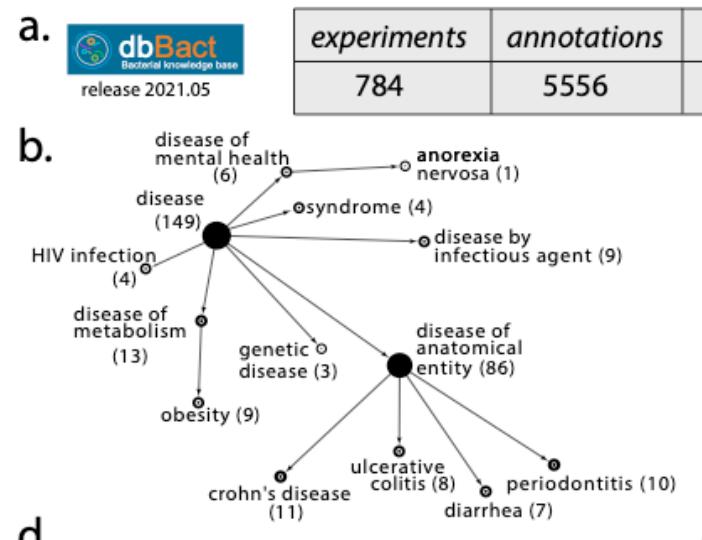

d.

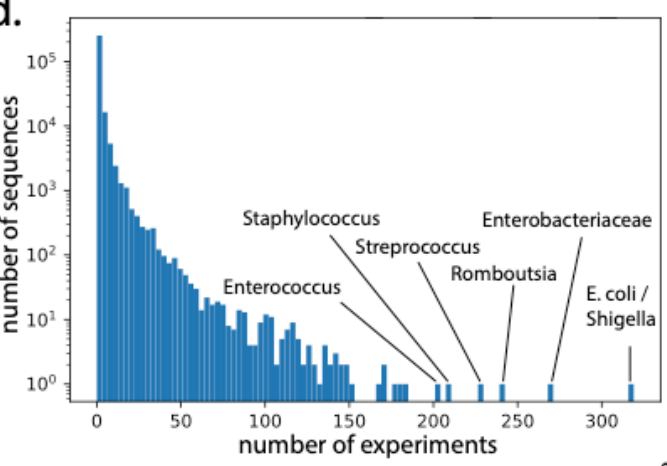

e.
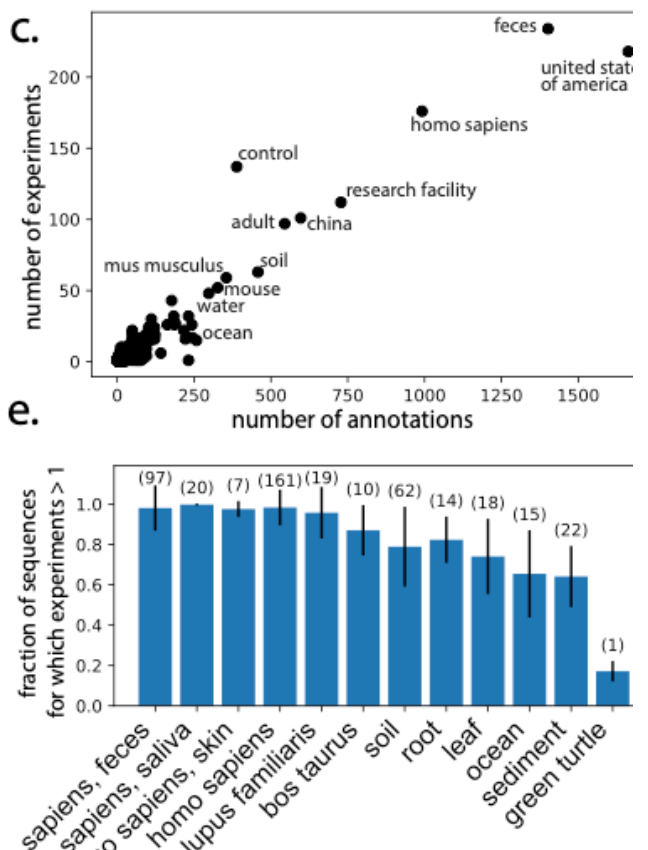

f.
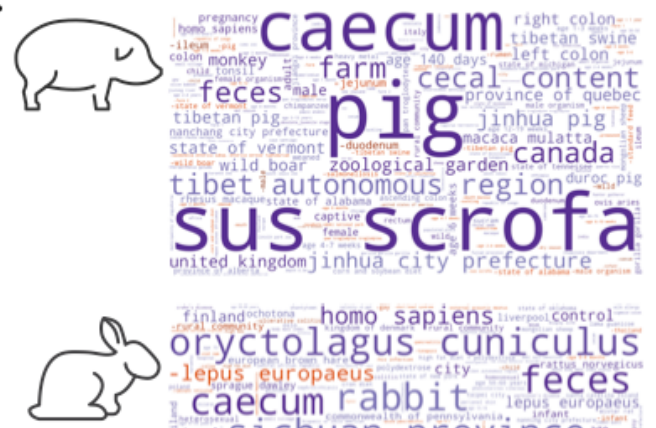

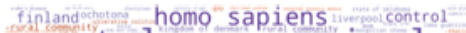
oryctolagus cuniculus caecum rabbit feces us sichuan province nature reserve prom col on european rabit adult $=$ Fi jiangsu province

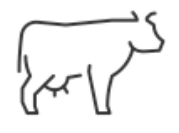

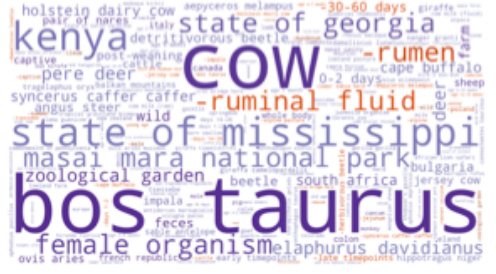

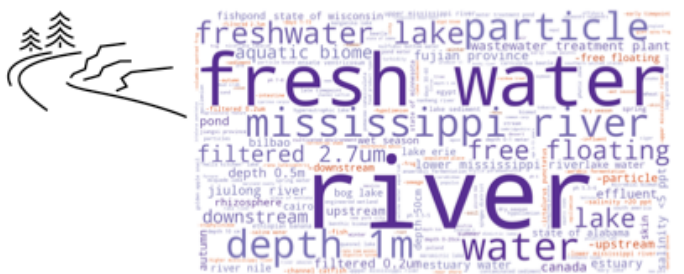

canis lupus familiaris italy
chicken f
child homo sapiens infant control colon

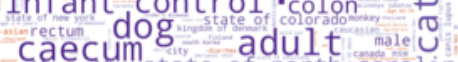
caecums adult ald

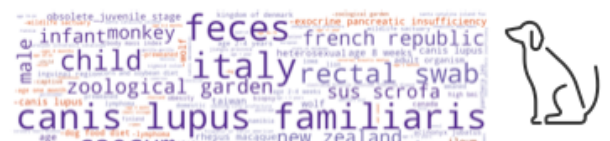
canis lupus familiaris kingdom of spainadult pigcolon controldog cat = homo sapiens rectum untelis catus state of minnesota

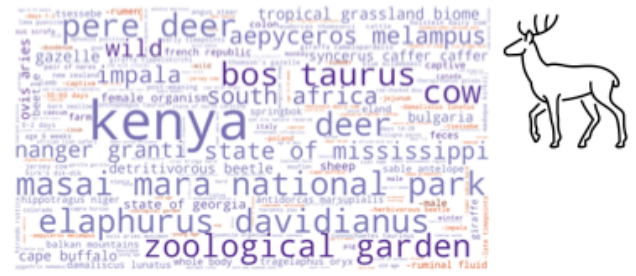

$a d u l t$ tsuting homo sapiens $\approx$

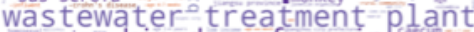
$f e c e s$ 
Figure 3: Knowledge base scope and comprehensiveness. a. Scope of dbBact release 2021.05 (used for the analysis presented in this paper). b. The number of experiments for representative disease categories based on the DOID ontology. c. Scatter plot of the total number of annotations and experiments in which each dbBact term appears. d. Histogram of the number of experiments in which each dbBact sequence appears. e. Knowledge base comprehensiveness. The fraction of "COMMON" sequences from each experiment that have been annotated in additional experiments is shown for various terms. The number of experiments containing the term is designated above each bar. f. Comprehensiveness in a source tracking task. Sequences from eight sample types from Hägglund et al. were blindly submitted to dbBact. Their word clouds clearly display the sources of the samples (shown by the matched cartoon). Term sizes correspond to the F-score of each term, combined for all sequences present in $>0.3$ of the samples (for each sample type).

\section{The advantage of sequence-based associations}

Results of 16S rRNA profiling experiments comprise a list of ASVs found in each sample and their abundances. Corroboration of these results with other microbiome studies is typically performed by searching published studies mentioning the taxonomy of these sequences. In many cases, however, such text-based mining may be limited because of constraints in taxonomic assignment. First, taxonomy is far from being full, e.g., species-level assignment is missing for about $80 \%$ of $16 \mathrm{~S}$ rRNA sequences in Greengenes (46), and about $35 \%$ of the Greengenes sequences lack a genus assignment (47). Second, in many cases the same assigned taxonomy may be associated with vastly different phenotypes. As observed by the Earth Microbiome Project, bacteria of the same genus may be present in vastly different habitats, whereas specific sequences are associated with a certain habitat (12). This phenomenon underscores the importance of sequence-based association as provided by dbBact. For example, both sequences in Figure $4 \mathrm{a}$ belong to the genus Blautia, hence taxonomy-based associations may conclude that they play similar "roles" and are associated with the same phenotype. But querying dbBact with each of these two sequences results in a strikingly different picture, which we refer to as a "good" and "bad" Blautia. The "good" Blautia is more abundant in healthy controls than in patients of type 1 diabetes (T1D), Crohns' disease (CD), inflammatory bowel disease (IBD), diarrhea, and kidney 
stones (Figure 4a), whereas the "bad" Blautia is more prevalent in patients suffering from IBD, $\mathrm{CD}$ and ulcerative colitis (Figure 4b).

Collecting all "disease" related dbBact annotations shows that the "bad" Blautia is "HIGHER IN" in the disease group (compared to controls) in 8/9 disease annotations associated with it, whereas the "good" Blautia is "LOWER IN" in the disease group (compared to controls) in 22/24 disease annotations (Figure 4c). Therefore, sequence-based analysis provides a solid genotype-tophenotype association compared to taxonomy-based associations.

a.

'good' Blautia

TACGTAGGGGGCAAGCGTTATCCGGATTTACTGGGTGTAAAGGGAGCGTAGACGGACTGG CAAGTCTGATGTGAAAGGCGGGGGCTCAACCCCTGGACTGCATGGAAACTGTTAGTCTTGAGTGCCG

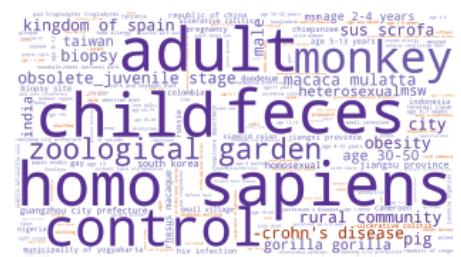

- HIGHER IN: control; LOWER IN: diabetes mellitus type i, diabetes mellitus; SOURCE: feces, homo sapiens, child, nigeria, obsolete juvenile stage

- HIGHER IN: control; LOWER IN: crohns disease, ulcerative colitis; SOURCE: feces, homo sapiens,

adult, united states of america, child, atlanta, obsolete juvenile stage, age $<17$ years

- HIGHER IN: healthy controls; LOWER IN: people hospitalized with diarrhea; SOURCE: feces,

homo sapiens, israel

- HIGHER IN: healthy controls; LOWER IN: nephrolithiasis; SOURCE: feces, homo sapiens, china,

adult, nanning city, prefecture age 50-60 years

- HIGHER IN: healthy donors; LOWER IN: kids with ibd; SOURCE: feces, homo sapiens, united

states of america, commonwealth of pennsylvania

- HIGHER IN: control; LOWER IN: irritable bowel syndrome; SOURCE: feces, homo sapiens,

kingdom of spain

... 200 annotations in tota b.

'bad'Blautia

TACGTAGGGGGCAAGCGTTATCCGGATTTACTGGGTGTAAAGGGAGCGTAGACGGAAGAG CAAGTCTGATGTGAAAGGCTGGGGCTTAACCCCAGGACTGCATTGGAAACTGTTTTTCTAGAGTGCCG
C.

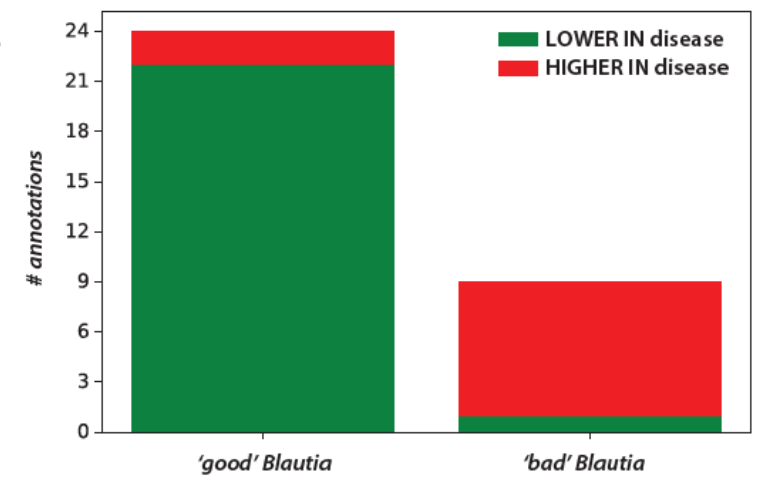

Figure 4: Taxonomy may be misleading. a. Two sequences of the genus Blautia, that differ by nine bases over the $150 \mathrm{bp}$ Illumina read of the $16 \mathrm{~S}$ rRNA V4 region are associated with opposite phenotypes, as discovered by dbBact. The two word clouds and annotations for each sequence, display "opposite" associations with disease. The left sequence is more prevalent in healthy subjects ("good" Blautia), whereas the other is highly abundant in a series of disease-related annotations. Such differences can be traced through dbBact, but are completely missed by a taxonomy-based analysis. b. The number of disease-related 
annotations for the two Blautia sequences across dbBact displays an opposite trend of being low and high in disease, for the "good" and "bad" Blautia, respectively. The total number of annotations in dbBact 2021.05 associated with the "good" and "bad" Blautia sequences is 377 and 124 , respectively.

\section{dbBact provides a pan-microbiome view: Detailed example}

dbBact may add another layer to data analysis in microbiome studies by identifying commonalities between different conditions and diseases, generating novel biological hypotheses. To demonstrate such a pan-microbiome analysis, we use data from a study comparing subjects consuming an American diet to a calorie restricted diet (48), and demonstrate the use of dbBact term enrichment. Fecal samples from two groups of lean individuals $(\mathrm{BMI}<25)$ who followed either an American diet (AMER) or a caloric restriction diet (CR) were selected. Standard analysis with FDR set to 0.1 (see "standard analysis" section in Methods) identified 28 and 141 bacterial sequences significantly more abundant in AMER and CR cohorts, respectively (Figure 3a). For clarity, we refer to these groups of sequences as S-AMER and S-CR, respectively. Figure $3 \mathrm{~b}$ shows the internal "transformation" performed by dbBact from a heatmap of bacterial abundances to a heatmap of association scores of terms for each sequence (columns in Figure 3a-b are aligned and correspond to the same sequences). For example, the term "high BMI," appears in almost all SCR sequences, while it is almost absent in S-AMER sequences. These association scores in Figure $3 \mathrm{~b}$ are then used as input to a non-parametric differential abundance test (see Methods section "statistical analysis in dbBact"), identifying terms significantly enriched in each of the two sequence groups (Figure 3c). Results indicate that sequences in the S-CR group are associated with terms related to low BMI ("low bmi," "LOWER IN high bmi") and with rural/undeveloped habitats ("LOWER IN united states of america," "small village") (see Supplementary File 4 for the full list of enriched terms). By contrast, bacteria from the S-AMER group have a significantly higher number of annotations related to high BMI ("LOWER IN low bmi," "high bmi") and urban/modernized habitats ("state of oklahoma," "LOWER IN rural community," "LOWER IN small village").

Thus, although participants from both diet groups were lean, certain aspects of the underlying microbiome were associated with high and low BMI bacteria, for AMER and CR, respectively. Additionally, bacteria enriched in CR vs. AMER tend to be associated with rural/undeveloped 
habitats, which may indicate an adaptation of some bacteria found in rural communities to a lowcalorie/higher vegetable diet content.

To further confirm the relationship between diet and BMI, we collected all sequences across dbBact having a "high bmi" annotation, resulting in 319 sequences. The overlap between these sequences and the S-AMER and S-CR groups is shown in Figure 3d (left). Although 93\% (26/28) of S-AMER sequences overlap with "high bmi"-associated sequences, the overlap of S-CR sequences is $11 \%(15 / 141)$, i.e., a much larger fraction of S-AMER sequences is associated with high BMI. An analogous Venn diagram for the term "low bmi" displays an overlap of 54\% (15/28) and 78\% (110/141) of S-AMER and S-CRON bacteria with "low bmi"-associated bacteria in dbBact, respectively (Figure 3d left). As participants from both CR and AMER groups were lean $(\mathrm{BMI}<25)$, one may hypothesize that the effect of BMI on the microbial composition, observed in various studies, is due to dietary differences rather than the high BMI phenotype.

Remark regarding spurious/irrelevant terms: dbBact release 2021.05 contains annotations of over 2500 unique terms, some appearing only in a few experiments. As a result, word clouds and bar plots may often include seemingly odd terms. For example, the term "state of oklahoma" in Figure $5 \mathrm{~d}$ is significantly enriched in S-AMER, a fact that seems implausible. This term appears only in two dbBact experiments, one of which compared a rural community in Peru to an urban community in Oklahoma (49). Hence, annotations from this experiment mentioned the term "state of oklahoma" together with more relevant terms (e.g., "rural community") which, in turn, caused its inclusion. As dbBact continues to grow, such "transient" irrelevant inductions are expected to diminish. 

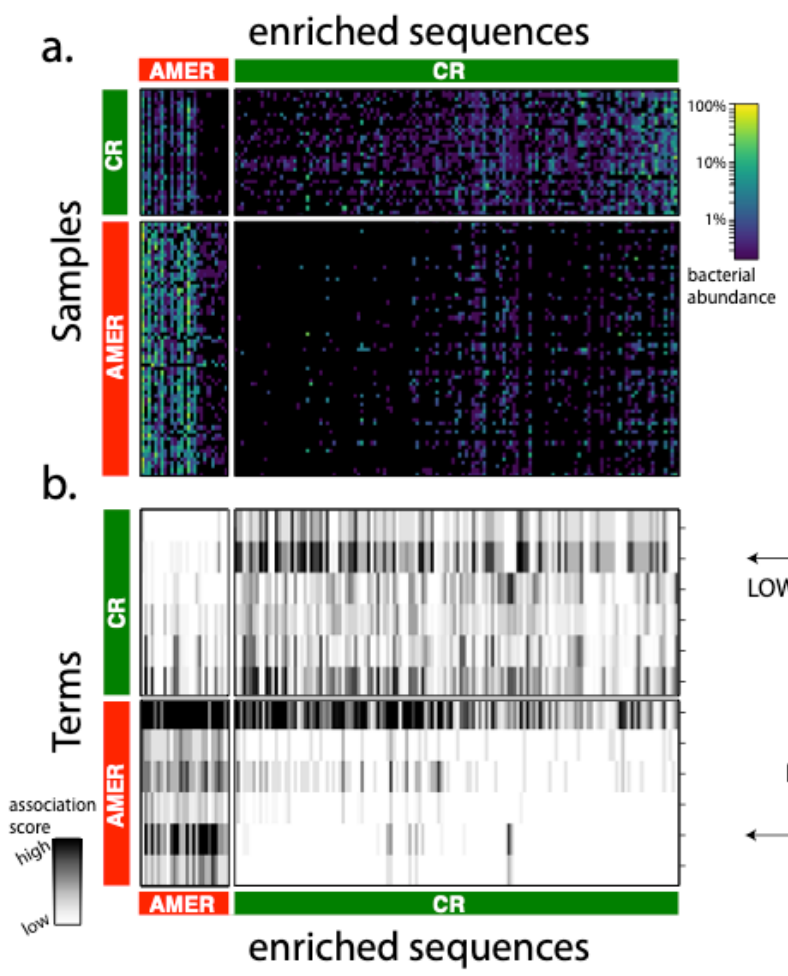

d.
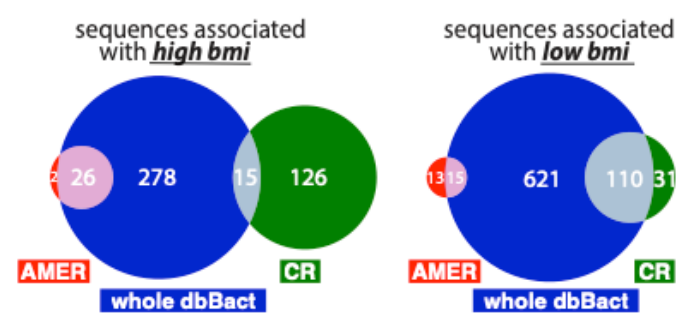

C.
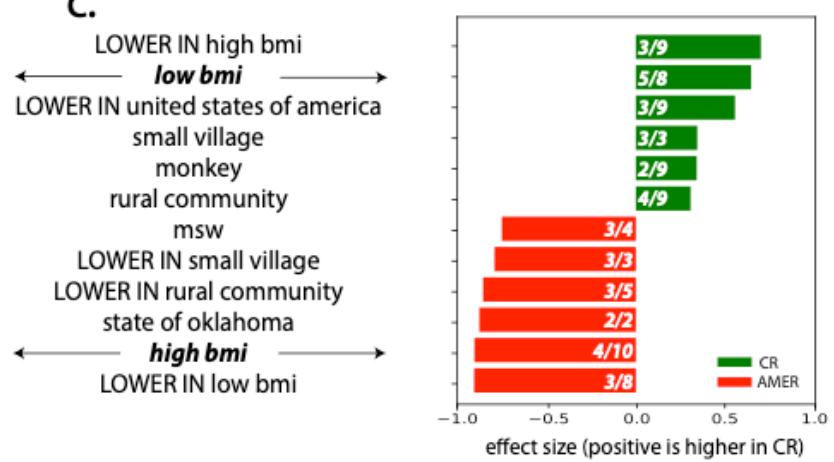

Figure 5. Linking caloric restriction associated bacteria to other phenotypes. a. Heatmap displaying bacterial abundances across fecal samples (rows) of low BMI individuals $(\mathrm{BMI}<25)$ practicing either a caloric restriction diet $(\mathrm{CR}, \mathrm{n}=33)$ or an American $\operatorname{diet}$ (AMER, $\mathrm{n}=66$ ), over a set of sequences (columns) that are significantly higher in either group. A differential abundance test (rank-mean test with dsFDR=0.1 multiple hypothesis correction) identified 136 bacteria higher in the CR group (S-CR) and 27 bacteria higher in the AMER group (S-AMER). b. dbBact terms (rows) enriched in the sequences appearing in panel $\mathbf{a}$ (columns in panels $\mathbf{a}$ and $\mathbf{b}$ are aligned). Heatmap values indicate the term score for each bacterium. Terms were identified using a non-parametric rank mean difference test with dsFDR=0.1 (top 6 terms for each direction are shown; see Supplementary File 4 for full list of enriched terms). c. Summary of the top enriched terms in the CR and AMER diets (green and red bars, respectively). Bar length and numbers are as in Figure 2. d. Venn diagrams of dbBact annotations related to the terms "low bmi" (right) and "high bmi" (left). Green and red circles indicate the number of sequences associated with the term in the CR and AMER diets, respectively; the blue circle indicates the number of such sequences across dbBact as a whole. The intersections of "low bmi" bacteria with the CR group are significantly higher ( $p=7 \mathrm{E}-5$, using two-sided Fisher's exact test), confirming the association. Similarly, the intersection of "high bmi" annotated sequences across dbBact 
with the AMER group is significantly higher than that with the CR group ( $p=3 \mathrm{E}-17$, using two-sided Fisher's exact test).

\section{dbBact allows formulating biological hypotheses}

This section provides two examples of biological hypotheses based on the re-analysis of published data using dbBact. Two additional examples appear in Supplementary Results and are briefly described following the example given below.

Fruit consumption in American Gut participants

Samples from the American Gut project (50) were filtered based on levels of fruit consumption (higher/lower than three fruits per week). Stratifying for age, gender and BMI resulted in 1071 samples in each group (see Methods section "Datasets processing" for details). Standard analysis yielded 25 sequences in each group, referred to as S-high and S-low, respectively. dbBact enrichment analysis (Figure 6a) shows that S-low sequences were associated with diets in industrialized regions (e.g., "LOWER IN rural community"), and S-high was enriched with the terms "hunter gatherer," "monkey," and rural environment-related terms. For example, the term "monkey," appearing 5093 times across dbBact, is associated with 84\% (21/25) of S-high sequences, compared to $16 \%$ (4/25) in S-low. This may indicate that the majority of bacteria positively affected by high fruit diet are universal responders to fruit consumption, and thus also appear in monkeys. Therefore, some of the differences in microbial communities between humans and monkeys are due to diet rather than host-microbe co-evolution.

Acute pancreatitis and Crohn's disease share gut bacteria

Fecal samples of 135 acute pancreatitis patients (seven days from the onset of symptoms) and of 35 healthy controls were analyzed (51). Standard analysis detected 39 sequences higher in pancreatitis patients and 300 sequences higher in healthy controls, referred to as S-panc and Shealthy, respectively. dbBact enrichment analysis (Figure 6b) shows that S-healthy sequences were enriched in health-associated terms such as "LOWER in crohn's disease" and "LOWER in ulcerative colitis," whereas enriched terms in S-panc sequences included disease associated terms such as "diarrhea" and "crohn's disease," as well as "LOWER in control." For example, 67\% (26/39) of the S-panc sequences were independently annotated as "crohn's disease"-associated sequences across dbBact, compared to only 13\% (40/300) of S-healthy. Therefore, dbBact hints at a common gut response to acute pancreatitis, diarrhea, and Crohn's disease, i.e., a phenomenon of general dysbiosis formerly suggested by Duvallet et al. (52). Next, we focused on the twelve 
sequences in S-panc that are not associated with either Crohn's disease or ulcerative colitis across dbBact annotations. The terms associated with these thirteen sequences include "fermentation," "homo sapiens," "feces," and "skin," as well as "soil" and sequences tagged as "candidate contaminants" (Figure S3 and Supplementary File 5). Additional experimental validation is required to determine whether some of these bacteria are pancreatitis-specific.

Two additional examples are presented in the Supplementary results section. In the first example, dbBact unravels the sources of air samples in an urban region during a dust storm and during ambient air (53). dbBact found that fecal bacteria from human and various farm animals were present during ambient weather conditions, whereas dust storms brought bacteria associated with "desert" and "soil." The second example analyzes microbial profiling of the skin swabs of bank voles. Samples were collected inside the uninhabited Chernobyl exclusion zone, and compared to those collected outside the contaminated region (54). dbBact analysis indicated that some of the differences in skin microbial communities of bank voles inhabiting the two regions may be attributed to differences in exposure to humans and farm animals, rather than to exposure to radioactivity. 


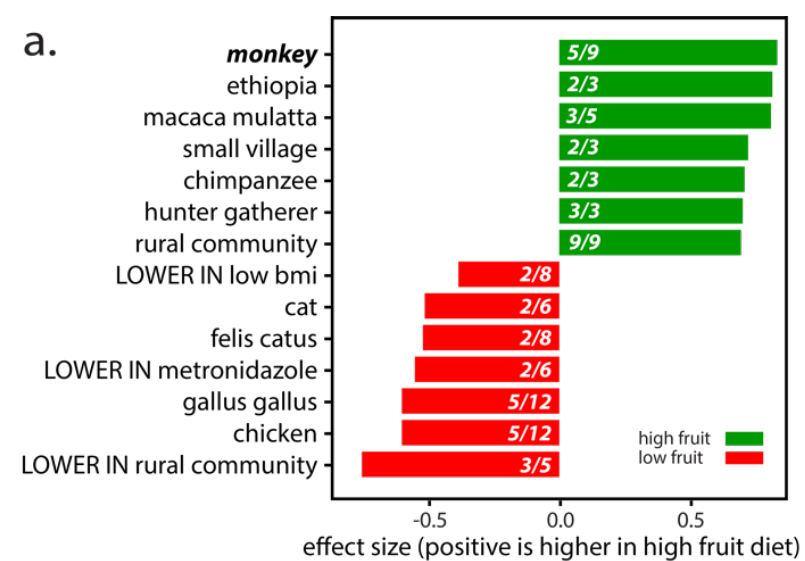

sequences associated with monkey

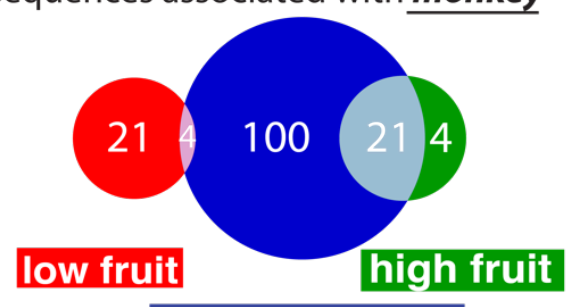

whole dbBact

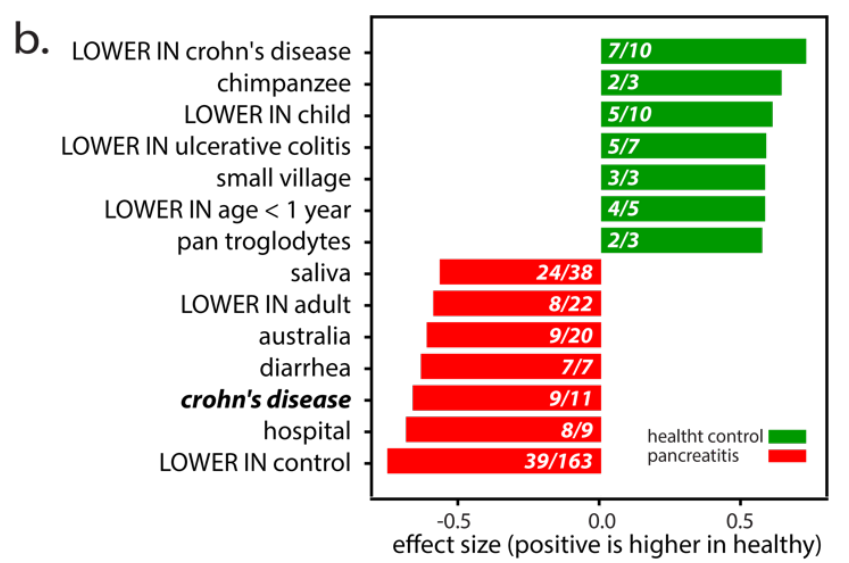

sequences associated with crohn's disease

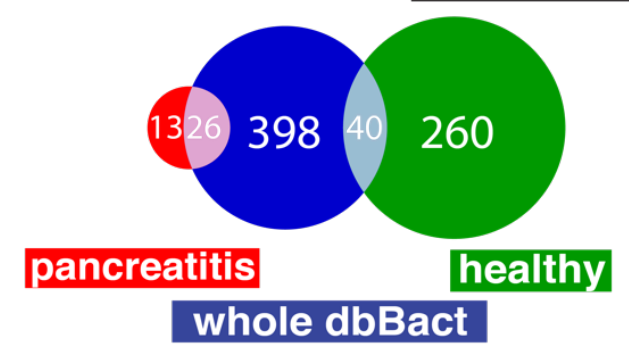

Fig 6. dbBact allows formulating biological hypotheses. Examples of pan-microbiome associations a. Fecal samples of American gut participants based on their fruit consumption. b. Fecal samples of acute pancreatitis vs. healthy controls. Panels display a bar plot of enriched terms in each group. Also shown is a Venn diagram for a representative term ("monkey" and "crohns" disease" in panels a and b, respectively), displaying the number of dbBact sequences associated with the term (blue circle), and their overlap with sequences associated with that term in either group (red and green circles). The numbers indicate the number of sequences in each part of the Venn diagrams.

\section{Discussion}

dbBact is a wiki-like bacterial knowledge base for collecting information from 16S rRNA amplicon studies. It creates a collaborative central repository suitable for subsequent analysis and it provides a pan-microbiome view.

An important advantage of dbBact, compared to standard meta-analysis methods, is that the latter may suffer from the "streetlight effect" (55). For example, when examining the effect of fruit consumption in the American Gut experiment (Fig 6a), one might consider including other dietrelated studies in the meta-analysis. But this would miss the link between high fruit consumption 
and primate-associated bacteria. dbBact retrieves annotations from a wide range of sample types and habitats, providing additional and potentially unexpected insights into the biological roles of bacteria.

Terms in dbBact annotations are based on ontologies, providing a common language for phenotype description. The tree structure of ontologies facilitates the discovery of commonalities between bacteria in studies conducted under similar, albeit not identical, conditions. For example, data from Crohn's disease and ulcerative colitis experiments may be combined based on their ontological "parent" term "inflammatory bowel disease." Moreover, many "cross-sectional" questions may be asked and possibly answered using dbBact. For instance, what terms are similar with respect to their bacteria (e.g., are dogs more similar to cats or to wolves?), or are there connections between phylogeny and specific phenotypes (e.g., does genus $\mathrm{X}$ appear only in host $\mathrm{Y}$ or in geographic location Z?).

Apart from putting forth novel hypotheses, dbBact makes possible the detection of sources of bacterial groups. We recommend querying dbBact as a first step in any microbiome analysis (e.g., using the interactive heatmap of the dbBact-Calour module). Identifying relevant bacterial groups and their dbBact annotations fosters an initial understanding of biological processes, supporting better downstream analysis. dbBact also enables associating bacteria with a "candidate reagent contaminant" annotation. We have encountered numerous cases where examining bacteria in a study detected contaminations, e.g., bacteria having mostly "mus musculus" annotations, although samples were of human origin.

The current coverage of dbBact is high in a large number of habitats, but many other habitats are still poorly covered (e.g., Figure 3e). Therefore, terms appearing in a small number of annotations may lead to dubious conclusions. For example, dbBact contains a single experiment originating from a scrubland environment. This experiment profiled the leaf microbiome of ivy plants, hence, querying a set of ivy leaf-related bacteria may result in the enrichment of both "ivy" and "scrubland" terms. Therefore, to avoid incorrect conclusions, users are advised to further examine the set of experiments associated with each enriched term. As the number and diversity of experiments in dbBact increase, such spurious terms are expected to be suppressed. Tens of 
microbiome studies are published weekly, but the dbBact team can process only a fraction. We expect the microbiome community to contribute to dbBact and help increase the number and diversity of uploaded studies.

dbBact may also be used in shotgun metagenomics studies. Whenever $16 \mathrm{~S}$ rRNA sequences are inferred from shotgun data they may be submitted as queries or uploaded to dbBact.

The "linking" mechanism for harmonizing studies from different variable regions enables shotgun and amplicon studies to be integrated into one coherent knowledge base. Similarly, studies using long read technologies (or synthetic long reads) also provide full-length 16S rRNA sequences, and thus can be integrated into dbBact in the same manner.

In sum, dbBact provides another layer of data analysis in microbiome studies, which we hope would be adopted by the community. Moreover, the scope and ontology-based structure of dbBact provides means for studying core factors affecting bacterial communities, possibly answering questions that could not have otherwise been asked.

\section{Acknowledgements}

We wish to thank Tzipi Brown and Rotem Hadar from the Sheba Microbiome Center, Zhenjiang (Zech) Xu, Jon Sanders, Qiyun Zhu, Tomasz Kosciolek, Stefan Janssen and Jeremiah Minich for fruitful discussions, feedback, and suggestions. N.S. is funded by the Ministry of Science, Technology \& Space, Israel (Grant 3-16033). 


\section{Methods}

As dbBact is constantly growing in scope, and to facilitate reproducibility, the dbBact infrastructure described in this section and all analyses presented in the paper carried out using dbBact release 2021-05-09, available for download as part of the weekly snapshots at https://dbbact.org/download.

\section{Implementation}

\section{Database}

The dbBact database is stored as a SQL relational database (PostgreSQL 9.5.10). The database schema and detailed table descriptions are provided in Supplementary File 1 and Figures S5-6.

\section{Ontologies}

Table S1 presents ontologies available in dbBact release 2021-05. dbBact supports the addition of ontologies to allow more accurate annotations. When users provide terms that do not appear in any of these ontologies, a new term is automatically added to the generic dbBact ontology.

\section{ASV sequences}

\section{Primers and trimming}

dbBact uses exact prefix search for sequence identification, and therefore all sequences in dbBact are primer trimmed and originate from one of the supported 16S rRNA forward primers. For dbBact release 2021.05, the supported forward primers are V1-27F (AGAGTTTGATCMTGGCTCAGxxx), V3-341F (CCTACGGGNGGCWGCAGxxx), V4-515F (GTGCCAGCMGCCGCGGTAAxxx), where " $x x x$ " denotes the beginning of the ASV sequence stored in dbBact. Although additional primers can be added to dbBact, the vast majority of $16 \mathrm{~S}$ rRNA studies uses one of the three primers described. The minimum length of sequences uploaded to dbBact is 100bp. Upon upload, sequences are stored at their full length rather than being truncated to a fixed length. When submitting a query sequence, exact sequence matches are searched using length=min(query_sequence_length, database_sequence_length). 


\section{Taxonomy assignment}

A python script runs daily to add taxonomy assignments to uploaded dbBact ASV sequences using RDP version 2.12 (56). Although taxonomy is not used in dbBact for analysis, it may be used for querying dbBact (e.g., retrieving annotations associated with bacteria of the genus Streptococcus).

\section{Inter-region querying}

dbBact supports the harmonization of microbiome studies performed using different protocols by inter-region linking. When submitting a sequence, dbBact uses the SILVA database of full length 16S rRNA genes (SILVA version 132, (34)) to identify sequences whose "footprint" in other variable regions matches the query. First, the SILVA sequences containing the query sequence are detected. Second, all dbBact sequences that match these SILVA sequences, in any region, are

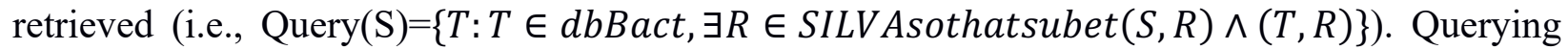
is performed using the "wholeSeqIDsTable" table in the dbBact implementation. To enable fast queries, a daily script is run on new dbBact sequences, linking all sequences sharing a SILVA sequence. Such linking is performed only when querying dbBact, therefore new versions of SILVA or other full length $16 \mathrm{~S}$ rRNA databases may be seamlessly applied. Currently, dbBact supports linking the V1, V3, and V4 forward primer reads; additional primers may be incorporated if needed.

\section{Queries of different sequence length}

Sequences uploaded to dbBact may vary in length depending on the sequencing platform and sequenced region. When adding new annotations, dbBact stores the full-length sequence of each ASV. For example, when two experiments provide information about the same bacterium using $150 \mathrm{bp}$ and $200 \mathrm{bp}$ reads, respectively, dbBact stores these sequences as separate entries and links each annotation to the corresponding sequence. Yet, when submitting a query using either sequence, dbBact retrieves annotations using exact match on the shortest common sequence, also retrieving annotations related to the other sequence. 


\section{dbBact interfaces}

\section{REST-API server}

The dbBact REST-API server (http://api.dbbact.org) is implemented in Python 3.6, using Flask version 0.12 /Gunicorn v19.9 to handle web queries, and psycopg2 version 2.7.1 for handling Postgres data queries. Full API documentation is available at http://api.dbbact.org/docs. Examples using the REST-API for querying are available at: https://github.com/amnona/dbbact-examples. The REST-API enables access to all dbBact functions. Querying dbBact or adding anonymous annotations does not require registration. Registration by username/password enables editing annotations submitted by the same user.

\section{dbBact website}

The dbBact website (http://dbbact.org) enables dbBact annotation retrieval based on ASVs, taxonomy, or ontology terms. Additionally, the website provides word-cloud generation and term enrichment analysis. The source code for the website as well as deployment instructions are available on the dbBact-website github page (https://github.com/amnona/dbbact-website).

dbBact-Calour interface

dbBact is integrated into the Calour microbiome analysis program (https://github.com/biocore/ calour), using the dbBact-Calour module (https://github.com/amnona/dbbact-calour). Using this interface, users can both query dbBact regarding bacterial sequences, and add new annotations. The dbBact-Calour module provides dbBact annotation retrieval from the interactive Calour heatmap display, showing all annotations associated with the selected sequence. Additionally, the module enables GUI-based creation of new dbBact annotations for selected sequences, and performs term enrichment analysis and word cloud generation. A Jupyter notebook tutorial is available at http://biocore.github.io/calour/notebooks/microbiome databases.html.

The module also works with EZCalour, the full GUI version of Calour, (https://github.com/amnona/EZCalour). A tutorial for dbBact enrichment analysis using EZCalour is available at: https://github.com/amnona/EZCalour/blob/master/using-ezcalour.pdf. 
Qiime2 plugin

The q2-dbBact plugin (https://github.com/amnona/q2-dbbact) enables dbBact annotation-based analysis using the qiime 2 framework (35). The interface provides term enrichment analysis for the output of various qiime2 differential abundance plugins (ANCOM (57), Songbird (58), ALDEx2 (59), DACOMP (60), or a rank-mean method). Additionally, the plugin supports dbBact term word cloud and interactive heatmap generation.

\section{Data availability}

Table 1 details the locations of different dbBact sites

\begin{tabular}{|l|l|}
\hline & Location \\
\hline dbBact website & $\underline{\text { http://dbbact.org }}$ \\
\hline dbBact website source code & $\underline{\text { https://github.com/amnona/dbbact-website }}$ \\
\hline dbBact REST-API server & $\underline{\text { api.dbbact.org }}$ \\
\hline Documentation for the REST-API & $\underline{\text { api.dbbact.org/docs }}$ \\
\hline Examples for using the REST-API interface & $\underline{\text { https://github.com/amnona/dbbact-examples }}$ \\
\hline Source code for the REST-API server & $\underline{\text { https://github.com/amnona/dbbact-server }}$ \\
\hline Jupyter notebooks for figures presented in the paper & $\underline{\text { https://github.com/amnona/dbbact-paper }}$ \\
\hline $\begin{array}{l}\text { Weekly dump of the complete dbBact database } \\
\text { (excluding user details) }\end{array}$ & $\underline{\text { https://dbbact.org/download }}$ \\
\hline
\end{tabular}

Table 1: Locations of dbBact sites

\section{Standard analysis: Default dbBact preprocessing of an experiment}

Although dbBact is a wiki-style knowledge base, the vast majority of annotations in dbBact release 2021.05 was added by the dbBact team. Studies were selected from published microbiome papers, and annotations were added following the re-processing of the experimental data, using a "standard" manual analysis pipeline as follows:

The raw data of each scientific paper (i.e., per-sample FASTA files and corresponding metadata) were downloaded using the provided accession (e.g., by SRA/ENA accession or Qiita (21) study ID). When data or metadata were not available, the authors were contacted and provided the missing data directly. When primer sequences were part of the reads, they were removed using a custom script (https://github.com/amnona/GetData). Subsequently, the Deblur pipeline (15) was applied to the reads of each sample (Deblur script version 1.1.0, using default parameters, https://github.com/biocore/deblur), resulting in a denoised biom table. 
This biom table, together with the per-sample metadata, were manually re-analyzed using Calour (36), to add annotations capturing biological conclusions arising from the study. Three types of predicates were sought:

(i) "DIFFERENTIAL:” To detect sets of sequences associated with relevant conditions (e.g., sick vs. healthy), sequences significantly enriched between two conditions were identified using a nonparametric permutation based rank-mean test, followed by multiple hypothesis correction using dsFDR (61) (usually set to 0.1 ). The test was performed by the calour.diff_abundance() function. Correlations with continuous metadata fields (e.g., BMI) were detected with a permutation-based Spearman test with dsFDR correction using calour.correlation(). In both cases, the set of sequences higher or lower in one condition than in the other were then annotated as "DIFFERENTIAL," i.e., "HIGHER IN" condition 1 and "LOWER IN" condition 2.

(ii) "COMMON"/“DOMINANT:" For each study, sequences present in more than half of the relevant samples were annotated as "COMMON." "DOMINANT" sequences were identified as sequences whose mean frequency in the relevant samples was higher than 0.01 . In studies containing samples from multiple sources (i.e., fecal and saliva samples, or samples from individuals from several countries or disease vs. healthy), a "COMMON"/"DOMINANT" annotation was added separately to each source subset.

(iii) “CONTAMINANT:” When a study contained a set of negative control (blank) samples, ASVs showing higher frequency in these controls than in the non-blank samples were manually annotated as a possible "CONTAMINANT."

Examples of the different predicates appear in Table S3.

Remark: Using the abovementioned pipeline is not a prerequisite for adding new annotations, and any denoising method followed by statistical analyses can be applied by users contributing to dbBact.

\section{Statistical analysis in dbBact}

Word cloud generation

Calculating a term's $\mathrm{F}_{1}$ score: Given a set of query sequences $S$, each dbBact term $t$ is assigned an $\mathrm{F}_{1}$ score, corresponding to the harmonic mean of precision and recall. For each sequence $s$ in $S$, we calculate the the fraction of s's annotations that contain the term $t$. The average of these 
values across $S$ provides the precision of $t$ on $S$. Similarly, for recall we calculate the fraction of $t$ 's annotations that is associated with each $s$. To suppress terms that appear in a small number of experiments, the total number of $t$ 's annotations (i.e., the denominator) is artificially increased by 1. The average of these values across $S$ provides the recall of $t$ on $S$.

Displaying a word cloud. The word cloud size of each term is proportional to its $\mathrm{F}_{1}$ score. If the term appears in "LOWER IN" annotations, its color is orange, otherwise it is blue. The brightness of each term represents the number of experiments containing the term, indicating the reliability of the term (white for a single experiment; ranging to dark blue/orange for $>=10$ experiments).

Word clouds were generated with the dbBact-Calour module for Calour (https://github.com/amnona/dbbact-calour) using the draw_wordcloud() function.

\section{Term enrichment analysis}

Given two sets of sequences, $S 1$ and $S 2$, we search for terms significantly enriched in either group using the following steps:

(a) Calculating an annotation-based score per sequence and term. Each annotation $a$ in dbBact is assigned a "weight" $w(a)$ according to its predicate. The predicates "COMMON," "CONTAMINATION," and "DIFFERENTIAL" are assigned a weight of 1 , and the predicates "DOMINANT" and "OTHER" are assigned a weight of 2 and 0.5, respectively. These weights are applied to calculate a score $S \operatorname{core}(s, t)$ for each term $t$ in dbBact and a sequence $s$ in either $S 1$ or $S 2$. The score sums the weights of all annotations involving $s$ and $t$. When $t$ appears as "LOWER IN" in the predicate "DIFFERENTIAL," a new term "not $t$ " is created and assigned a weight of 1.

(b) Calculating effect size of a term. For a term $t, \operatorname{Score}(s, t)$ is calculated for all sequences in $S 1$ and $S 2$, and the effect size of $t$ is defined as

$$
e(t)=2\left|\left(\frac{\sum_{s \in S 1} \operatorname{Score}(s, t)}{|S 1|}-\frac{\sum_{s \in S 2} \operatorname{Score}(s, t)}{|S 2|}\right)\right| /(|S 1|+|S 2|)
$$

where $|S|$ corresponds to the number of sequences in the set $S$.

(c) Finding significant terms. Each term is assigned a p-value by comparing its scores over 1000 random permutations of the $S 1$ and $S 2$ sequences to sets of size $|S 1|$ and $|S 2|$. Subsequently, a dsFDR multiple hypothesis correction (with a threshold of 0.1) is applied to detect significant terms. 
(d) Calculating the significance of a term across experiments. Until this stage, we measured the enrichment of a term based on all dbBact experiments combined. To estimate whether such significance occurs across multiple experiments, or whether it is driven by a single or a few experiments, steps (a)-(c) were also repeated using each individual experiment that contains $t$. The total number of experiments containing each term and the fraction in which the term was significant appear in each figure.

The abovementioned analysis is performed using the dbbact-calour enrichment() function.

\section{Venn diagrams}

Given two sets of sequences, $S 1$ and $S 2$, and a term $t$, we plot a Venn diagram indicating the number of sequences associated with $t$ across all dbBact annotations, and the overlap of these sequences with $S 1$ and $S 2$.

\section{Dataset processing}

All datasets discussed in the paper were processed using the following pipeline: raw reads were downloaded and denoised using the Deblur pipeline (15) with default parameters; the resulting denoised biom table was loaded into Calour (36), and differentially abundant bacteria were identified using a permutation-based non-parametric rank mean test with dsFDR multiple hypothesis correction (61) set to 0.1 (using the calour diff_abundance() method). In the case of the American Gut Project dataset, multiple samples originating from the same individual were aggregated to a single sample using mean frequency for each ASV. The groups of high and low fruit consumption were controlled for confounders by stratifying samples in both groups based on the AGP metadata fields: age category (“AGE_CAT"), sex and BMI category ("BMI_CAT"), and randomly dropping samples to equalize the number of samples from each stratum prior to differential abundance testing. Term enrichment was performed using the Calour enrichment() method with default parameters $(\mathrm{dsFDR}=0.1)$ against dbBact release 2021.05. Accession numbers for each dataset used are available in Supplementary File 2. Jupyter notebooks used for the creation of each figure are available at https://github.com/amnona/dbbact-paper. 


\section{References}

1. Smil V. 2003. The Earth's biosphere: Evolution, dynamics, and change. MIT Press.

2. Pace NR. 1997. A molecular view of microbial diversity and the biosphere. Science (80- ) 276:734-740.

3. Hamady M, Walker JJ, Harris JK, Gold NJ, Knight R. 2008. Error-correcting barcoded primers for pyrosequencing hundreds of samples in multiplex. Nat Methods 5:235-237.

4. Herlemann DPR, Labrenz M, Jürgens K, Bertilsson S, Waniek JJ, Andersson AF. 2011. Transitions in bacterial communities along the $2000 \mathrm{~km}$ salinity gradient of the Baltic Sea. ISME J 5:1571-1579.

5. Bahram M, Hildebrand F, Forslund SK, Anderson JL, Soudzilovskaia NA, Bodegom PM, Bengtsson-Palme J, Anslan S, Coelho LP, Harend H, Huerta-Cepas J, Medema MH, Maltz MR, Mundra S, Olsson PA, Pent M, Põlme S, Sunagawa S, Ryberg M, Tedersoo L, Bork P. 2018. Structure and function of the global topsoil microbiome. Nature 560:233-237.

6. Peiffer JA, Spor A, Koren O, Jin Z, Tringe SG, Dangl JL, Buckler ES, Ley RE. 2013. Diversity and heritability of the maize rhizosphere microbiome under field conditions. Proc Natl Acad Sci U S A 110:6548-6553.

7. Muegge BD, Kuczynski J, Knights D, Clemente JC, González A, Fontana L, Henrissat B, Knight R, Gordon JI. 2011. Diet drives convergence in gut microbiome functions across mammalian phylogeny and within humans. Science 332:970-4.

8. Goodrich JK, Waters JL, Poole AC, Sutter JL, Koren O, Blekhman R, Beaumont M, Van Treuren W, Knight R, Bell JT, Spector TD, Clark AG, Ley RE. 2014. Human genetics shape the gut microbiome. Cell 159:789-799.

9. Mcdonald D, Hyde E, Debelius JW, Morton JT, Gonzalez A, Ackermann G, Aksenov AA, Behsaz B, Brennan C, Chen Y, Goldasich D, Dorrestein PC, Dunn RR, Fahimipour AK, Gaffney J, Gilbert JA, Gogul G, Green JL, Hugenholtz P, Humphrey G, Huttenhower C, Jackson MA, Janssen S, Jeste D V, Jiang L, Kelley ST, Knights D, Kosciolek T, Ladau J, Leach J, Marotz C, Meleshko D, Melnik A V, Metcalf JL, Mohimani H, Montassier E, Rahnavard G, Robbins-pianka A, Sangwan N, Shorenstein J, Smarr L, Vázquez-baeza Y, Vrbanac A, Wischmeyer P, Wolfe E, Zhu Q, Gut A, Jt M, Gonzalez A, Ackermann G, Behsaz B, Brennan C, Chen Y, Goldasich L, Pc D, Rr D, JI G, Hugenholtz P, Humphrey G, Huttenhower C, Ma J, Janssen S, Jiang L, St K, Knights D, Kosciolek T. 2018. American Gut : an Open Platform for Citizen Science Microbiome Research. mSystems 3:1-28.

10. Huttenhower C, Gevers D, Knight R, Abubucker S, Badger JH, Chinwalla AT, Creasy HH, Earl AM, Fitzgerald MG, Fulton RS, Giglio MG, Hallsworth-Pepin K, Lobos EA, Madupu R, Magrini V, Martin JC, Mitreva M, Muzny DM, Sodergren EJ, Versalovic J, Wollam AM, Worley KC, Wortman JR, Young SK, Zeng Q, Aagaard KM, Abolude OO, Allen-Vercoe E, Alm EJ, Alvarado L, Andersen GL, Anderson S, Appelbaum E, Arachchi HM, Armitage G, Arze CA, Ayvaz T, Baker CC, Begg L, Belachew T, Bhonagiri V, Bihan M, Blaser MJ, Bloom T, Bonazzi V, Paul Brooks J, Buck GA, Buhay CJ, Busam DA, Campbell JL, Canon SR, Cantarel BL, Chain PSG, Chen IMA, Chen L, Chhibba S, Chu K, Ciulla DM, Clemente JC, Clifton SW, Conlan S, Crabtree J, Cutting MA, Davidovics NJ, Davis CC, Desantis 
TZ, Deal C, Delehaunty KD, Dewhirst FE, Deych E, Ding Y, Dooling DJ, Dugan SP, Michael Dunne W, Scott Durkin A, Edgar RC, Erlich RL, Farmer CN, Farrell RM, Faust K, Feldgarden M, Felix VM, Fisher S, Fodor AA, Forney LJ, Foster L, Di Francesco V, Friedman J, Friedrich DC, Fronick CC, Fulton LL, Gao H, Garcia N, Giannoukos G, Giblin C, Giovanni MY, Goldberg JM, Goll J, Gonzalez A, Griggs A, Gujja S, Kinder Haake S, Haas BJ, Hamilton HA, Harris EL, Hepburn TA, Herter B, Hoffmann DE, Holder ME, Howarth C, Huang KH, Huse SM, Izard J, Jansson JK, Jiang H, Jordan C, Joshi V, Katancik JA, Keitel WA, Kelley ST, Kells C, King NB, Knights D, Kong HH, Koren O, Koren S, Kota KC, Kovar CL, Kyrpides NC, La Rosa PS, Lee SL, Lemon KP, Lennon N, Lewis CM, Lewis L, Ley RE, Li K, Liolios K, Liu B, Liu Y, Lo CC, Lozupone CA, Dwayne Lunsford R, Madden T, Mahurkar AA, Mannon PJ, Mardis ER, Markowitz VM, Mavromatis K, McCorrison JM, McDonald D, McEwen J, McGuire AL, McInnes P, Mehta T, Mihindukulasuriya KA, Miller JR, Minx PJ, Newsham I, Nusbaum C, Oglaughlin M, Orvis J, Pagani I, Palaniappan K, Patel SM, Pearson M, Peterson J, Podar M, Pohl C, Pollard KS, Pop M, Priest ME, Proctor LM, Qin X, Raes J, Ravel J, Reid JG, Rho M, Rhodes R, Riehle KP, Rivera MC, Rodriguez-Mueller B, Rogers YH, Ross MC, Russ C, Sanka RK, Sankar P, Fah Sathirapongsasuti J, Schloss JA, Schloss PD, Schmidt TM, Scholz M, Schriml L, Schubert AM, Segata N, Segre JA, Shannon WD, Sharp RR, Sharpton TJ, Shenoy N, Sheth NU, Simone GA, Singh I, Smillie CS, Sobel JD, Sommer DD, Spicer P, Sutton GG, Sykes SM, Tabbaa DG, Thiagarajan M, Tomlinson CM, Torralba M, Treangen TJ, Truty RM, Vishnivetskaya TA, Walker J, Wang L, Wang Z, Ward D V., Warren W, Watson MA, Wellington C,

Wetterstrand KA, White JR, Wilczek-Boney K, Wu Y, Wylie KM, Wylie T, Yandava C, Ye L, Ye Y, Yooseph S, Youmans BP, Zhang L, Zhou Y, Zhu Y, Zoloth L, Zucker JD, Birren BW, Gibbs RA, Highlander SK, Methé BA, Nelson KE, Petrosino JF, Weinstock GM, Wilson RK, White O. 2012. Structure, function and diversity of the healthy human microbiome. Nature 486:207-214.

11. Burcham ZM, Garneau NL, Comstock SS, Tucker RM, Knight R, Metcalf JL, Miranda A, Reinhart B, Meyers D, Woltkamp D, Boxer E, Hutchens J, Kim K, Archer M, McAteer M, Huss P, Defonseka R, Stahle S, Babu S, Nuessle T, Schowinsky V, Covert W, Truman W, Reusser W. 2020. Patterns of Oral Microbiota Diversity in Adults and Children: A Crowdsourced Population Study. Sci Rep 10:1-15.

12. Thompson LR, Sanders JG, Mcdonald D, Amir A, Ladau J, Locey KJ, Prill RJ, Tripathi A, Gibbons SM, Ackermann G, Navas-molina JA, Janssen S, Kopylova E, Vázquez-baeza Y, González A, Morton JT, Mirarab S, Xu ZZ, Jiang L. 2017. A communal catalogue reveals Earth' s multiscale microbial diversity.

13. Federhen S. 2012. The NCBI Taxonomy database. Nucleic Acids Res.

14. Parr CS, Wilson N, Leary P, Schulz KS, Lans K, Walley L, Hammock JA, Goddard A, Rice J, Studer M, Holmes JTG, Corrigan RJ. 2014. The Encyclopedia of Life v2: Providing Global Access to Knowledge About Life on Earth. Biodivers Data J 2.

15. Amir A, McDonald D, Navas-Molina JA, Kopylova E, Morton JT, Xu ZZ, Knightly EP, Thompson LR, Hyde ER, Gonzalez A, Knight R. 2017. Deblur Rapidly Resolves Single-Nucleotide Community Sequence Patterns 2:1-7.

16. Callahan BJ, McMurdie PJ, Rosen MJ, Han AW, Johnson AJA, Holmes SP. 2016. DADA2: High-resolution sample inference from Illumina amplicon data. Nat Methods 
13:581-3.

17. Edgar R. 2016. UNOISE2: improved error-correction for Illumina 16S and ITS amplicon sequencing. bioRxiv 081257.

18. Prodan A, Tremaroli V, Brolin H, Zwinderman AH, Nieuwdorp M, Levin E. 2020. Comparing bioinformatic pipelines for microbial 16S rRNA amplicon sequencing. PLoS One 15:1-19.

19. Dennis G, Sherman BT, Hosack DA, Yang J, Gao W, Lane HC, Lempicki RA. 2003. DAVID: Database for Annotation, Visualization, and Integrated Discovery. Genome Biol 4.

20. Raudvere U, Kolberg L, Kuzmin I, Arak T, Adler P, Peterson H, Vilo J. 2019. G:Profiler: A web server for functional enrichment analysis and conversions of gene lists (2019 update). Nucleic Acids Res 47:W191-W198.

21. Gonzalez A, Navas-Molina JA, Kosciolek T, McDonald D, Vázquez-Baeza Y, Ackermann G, DeReus J, Janssen S, Swafford AD, Orchanian SB, Sanders JG, Shorenstein J, Holste H, Petrus S, Robbins-Pianka A, Brislawn CJ, Wang M, Rideout JR, Bolyen E, Dillon M, Caporaso JG, Dorrestein PC, Knight R. 2018. Qiita: rapid, web-enabled microbiome meta-analysis. Nat Methods 15:796-798.

22. Proctor LM, Creasy HH, Fettweis JM, Lloyd-Price J, Mahurkar A, Zhou W, Buck GA, Snyder MP, Strauss JF, Weinstock GM, White O, Huttenhower C. 2019. The Integrative Human Microbiome Project. Nature 569:641-648.

23. Mitchell AL, Almeida A, Beracochea M, Boland M, Burgin J, Cochrane G, Crusoe MR, Kale V, Potter SC, Richardson LJ, Sakharova E, Scheremetjew M, Korobeynikov A, Shlemov A, Kunyavskaya O, Lapidus A, Finn RD. 2020. MGnify: The microbiome analysis resource in 2020. Nucleic Acids Res 48:D570-D578.

24. Parente E, De Filippis F, Ercolini D, Ricciardi A, Zotta T. 2019. Advancing integration of data on food microbiome studies: FoodMicrobionet 3.1, a major upgrade of the FoodMicrobionet database. Int J Food Microbiol 305.

25. McDonald D, Kaehler B, Gonzalez A, DeReus J, Ackermann G, Marotz C, Huttley G, Knight R. 2019. redbiom: a Rapid Sample Discovery and Feature Characterization System. mSystems 4:1-5.

26. Yao G, Zhang W, Yang M, Yang H, Wang J, Zhang H, Wei L, Xie Z, Li W. 2020. MicroPhenoDB associates metagenomic data with pathogenic microbes, microbial core genes, and human disease phenotypes. bioRxiv 2020.07.29.221010.

27. Cheng L, Qi C, Zhuang H, Fu T, Zhang X. 2020. GutMDisorder: A comprehensive database for dysbiosis of the gut microbiota in disorders and interventions. Nucleic Acids Res 48:D554-D560.

28. Janssens Y, Nielandt J, Bronselaer A, Debunne N, Verbeke F, Wynendaele E, Van Immerseel F, Vandewynckel YP, De Tré G, De Spiegeleer B. 2018. Disbiome database: Linking the microbiome to disease. BMC Microbiol 18:4-9.

29. Skoufos G, Kardaras FS, Alexiou A, Kavakiotis I, Lambropoulou A, Kotsira V, Tastsoglou S, Hatzigeorgiou AG. 2021. Peryton: A manual collection of experimentally supported microbe-disease associations. Nucleic Acids Res 49:D1328-D1333.

30. Waldron L, Jones H, Geistlinger L, Davis S, Huttenhower C, Segata N. 2021. https://bugsigdb.org/.

31. Yang J, Park J, Park S, Baek I, Chun J. 2019. Introducing murine microbiome database (MMDB): A curated database with taxonomic profiling of the healthy mouse 
gastrointestinal microbiome. Microorganisms 7.

32. Moitinho-Silva L, Nielsen S, Amir A, Gonzalez A, Ackermann GL, Cerrano C, Astudillo-Garcia C, Easson C, Sipkema D, Liu F, Steinert G, Kotoulas G, McCormack GP, Feng G, Bell JJ, Vicente J, Björk JR, Montoya JM, Olson JB, Reveillaud J, Steindler L, Pineda MC, Marra M V., Ilan M, Taylor MW, Polymenakou P, Erwin PM, Schupp PJ, Simister RL, Knight R, Thacker RW, Costa R, Hill RT, Lopez-Legentil S, Dailianis T, Ravasi T, Hentschel U, Li Z, Webster NS, Thomas T. 2017. The sponge microbiome project. Gigascience 6:1-7.

33. Schriml LM, Mitraka E, Munro J, Tauber B, Schor M, Nickle L, Felix V, Jeng L, Bearer C, Lichenstein R, Bisordi K, Campion N, Hyman B, Kurland D, Oates CP, Kibbey S, Sreekumar P, Le C, Giglio M, Greene C. 2019. Human Disease Ontology 2018 update: Classification, content and workflow expansion. Nucleic Acids Res.

34. Quast C, Pruesse E, Yilmaz P, Gerken J, Schweer T, Yarza P, Peplies J, Glöckner FO. 2013. The SILVA ribosomal RNA gene database project: Improved data processing and web-based tools. Nucleic Acids Res 41.

35. Bolyen E, Rideout JR, Dillon MR, Bokulich NA, Abnet CC, Al-Ghalith GA, Alexander H, Alm EJ, Arumugam M, Asnicar F, others. 2019. Reproducible, interactive, scalable and extensible microbiome data science using QIIME 2. Nat Biotechnol 37:852-857.

36. Xu ZZ, Amir A, Sanders JG, Zhu Q, Morton JT, Bletz MC, Tripathi A, Huang S, McDonald D, Jiang L, Knight R. 2019. Calour: an Interactive, Microbe-Centric Analysis Tool 4:1-12.

37. Ijaz UZ, Quince C, Hanske L, Loman N, Calus ST, Bertz M, Edwards CA, Gaya DR, Hansen R, McGrogan P, Russell RK, Gerasimidis K. 2017. The distinct features of microbial "dysbiosis" of Crohn's disease do not occur to the same extent in their unaffected, genetically-linked kindred. PLoS One.

38. Buttigieg PL, Pafilis E, Lewis SE, Schildhauer MP, Walls RL, Mungall CJ. 2016. The environment ontology in 2016: Bridging domains with increased scope, semantic density, and interoperation. J Biomed Semantics.

39. Buttigieg PL, Morrison N, Smith B, Mungall CJ, Lewis SE. 2013. The environment ontology: Contextualising biological and biomedical entities. J Biomed Semantics.

40. Whetzel PL, Noy NF, Shah NH, Alexander PR, Nyulas C, Tudorache T, Musen MA. 2011. BioPortal: Enhanced functionality via new Web services from the National Center for Biomedical Ontology to access and use ontologies in software applications. Nucleic Acids Res.

41. Mungall CJ, Torniai C, Gkoutos G V., Lewis SE, Haendel MA. 2012. Uberon, an integrative multi-species anatomy ontology. Genome Biol.

42. Malone J, Holloway E, Adamusiak T, Kapushesky M, Zheng J, Kolesnikov N, Zhukova A, Brazma A, Parkinson H. 2010. Modeling sample variables with an Experimental Factor Ontology. Bioinformatics.

43. Zmora N, Zilberman-Schapira G, Suez J, Mor U, Dori-Bachash M, Bashiardes S, Kotler E, Zur M, Regev-Lehavi D, Brik RBZ, Federici S, Cohen Y, Linevsky R, Rothschild D, Moor AE, Ben-Moshe S, Harmelin A, Itzkovitz S, Maharshak N, Shibolet O, Shapiro H, Pevsner-Fischer M, Sharon I, Halpern Z, Segal E, Elinav E. 2018. Personalized Gut Mucosal Colonization Resistance to Empiric Probiotics Is Associated with Unique Host and Microbiome Features. Cell. 
44. Amir A, McDonald D, Navas-Molina JA, Debelius J, Morton JT, Hyde E, Robbinspianka A. 2017. Correcting for Microbial Blooms in Fecal Samples during RoomTemperature Shipping Amnon. mSystems 2:1-5.

45. Hägglund M, Bäckman S, Macellaro A, Lindgren P, Borgmästars E, Jacobsson K, Dryselius R, Stenberg P, Sjödin A, Forsman M, Ahlinder J. 2018. Accounting for bacterial overlap between raw water communities and contaminating sources improves the accuracy of signature-based microbial source tracking. Front Microbiol.

46. DeSantis TZ, Hugenholtz P, Larsen N, Rojas M, Brodie EL, Keller K, Huber T, Dalevi D, Hu P, Andersen GL. 2006. Greengenes, a chimera-checked 16S rRNA gene database and workbench compatible with ARB. Appl Environ Microbiol.

47. Nejman D, Fuks G, Livyatan I, Rotter A, Shental N, Straussman R. 2019. The tumor microbiome: a pan-cancer perspective.

48. Griffin NW, Ahern PP, Cheng J, Heath AC, Ilkayeva O, Newgard CB, Fontana L, Gordon JI. 2017. Prior Dietary Practices and Connections to a Human Gut Microbial Metacommunity Alter Responses to Diet Interventions. Cell Host Microbe 21:84-96.

49. Obregon-Tito AJ, Tito RY, Metcalf J, Sankaranarayanan K, Clemente JC, Ursell LK, Zech Xu Z, Van Treuren W, Knight R, Gaffney PM, Spicer P, Lawson P, Marin-Reyes L, Trujillo-Villarroel O, Foster M, Guija-Poma E, Troncoso-Corzo L, Warinner C, Ozga AT, Lewis CM. 2015. Subsistence strategies in traditional societies distinguish gut microbiomes. Nat Commun.

50. McDonald D, Hyde E, Debelius JW, Morton JT, Gonzalez A, Ackermann G, Aksenov AA, Behsaz B, Brennan C, Chen Y, DeRight Goldasich L, Dorrestein PC, Dunn RR, Fahimipour AK, Gaffney J, Gilbert JA, Gogul G, Green JL, Hugenholtz P, Humphrey G, Huttenhower C, Jackson MA, Janssen S, Jeste D V., Jiang L, Kelley ST, Knights D, Kosciolek T, Ladau J, Leach J, Marotz C, Meleshko D, Melnik A V., Metcalf JL, Mohimani H, Montassier E, Navas-Molina J, Nguyen TT, Peddada S, Pevzner P, Pollard KS, Rahnavard G, Robbins-Pianka A, Sangwan N, Shorenstein J, Smarr L, Song SJ, Spector T, Swafford AD, Thackray VG, Thompson LR, Tripathi A, Vázquez-Baeza Y, Vrbanac A, Wischmeyer P, Wolfe E, Zhu Q, Knight R, Mann AE, Amir A, Frazier A, Martino C, Lebrilla C, Lozupone C, Lewis CM, Raison C, Zhang C, Lauber CL, Warinner C, Lowry CA, Callewaert C, Bloss C, Willner D, Galzerani DD, Gonzalez DJ, Mills DA, Chopra D, Gevers D, Berg-Lyons D, Sears DD, Wendel D, Lovelace E, Pierce E, TerAvest E, Bolyen E, Bushman FD, Wu GD, Church GM, Saxe G, Holscher HD, Ugrina I, German JB, Caporaso JG, Wozniak JM, Kerr J, Ravel J, Lewis JD, Suchodolski JS, Jansson JK, Hampton-Marcell JT, Bobe J, Raes J, Chase JH, Eisen JA, Monk J, Clemente JC, Petrosino J, Goodrich J, Gauglitz J, Jacobs J, Zengler K, Swanson KS, Lewis K, Mayer K, Bittinger K, Dillon L, Zaramela LS, Schriml LM, Dominguez-Bello MG, Jankowska MM, Blaser M, Pirrung M, Minson M, Kurisu M, Ajami N, Gottel NR, Chia N, Fierer N, White O, Cani PD, Gajer P, Strandwitz P, Kashyap P, Dutton R, Park RS, Xavier RJ, Mills RH, Krajmalnik-Brown R, Ley R, Owens SM, Klemmer S, Matamoros S, Mirarab S, Moorman S, Holmes S, Schwartz T, Eshoo-Anton TW, Vigers T, Pandey V, Treuren W Van, Fang X, Zech Xu Z, Jarmusch A, Geier J, Reeve N, Silva R, Kopylova E, Nguyen D, Sanders K, Salido Benitez RA, Heale AC, Abramson M, Waldispühl J, Butyaev A, Drogaris C, Nazarova E, Ball M, Gunderson B. 2018. American Gut: an Open Platform for Citizen Science Microbiome Research. mSystems. 
51. Zhu Y, He C, Li X, Cai Y, Hu J, Liao Y, Zhao J, Xia L, He W, Liu L, Luo C, Shu X, Cai Q, Chen Y, Lu N. 2018. Gut microbiota dysbiosis worsens the severity of acute pancreatitis in patients and mice. J Gastroenterol.

52. Duvallet C, Gibbons SM, Gurry T, Irizarry RA, Alm EJ. 2017. Meta-analysis of gut microbiome studies identifies disease-specific and shared responses. Nat Commun 8.

53. Gat D, Mazar Y, Cytryn E, Rudich Y. 2017. Origin-Dependent Variations in the Atmospheric Microbiome Community in Eastern Mediterranean Dust Storms. Environ Sci Technol.

54. Lavrinienko A, Tukalenko E, Mappes T, Watts PC. 2018. Skin and gut microbiomes of a wild mammal respond to different environmental cues. Microbiome 6:209.

55. Freedman DH. 2010. Why Scientific Studies Are So Often Wrong: The Streetlight Effect. Discover 31:55-57.

56. Wang Q, Garrity GM, Tiedje JM, Cole JR. 2007. Naïve Bayesian classifier for rapid assignment of rRNA sequences into the new bacterial taxonomy. Appl Environ Microbiol 73:5261-5267.

57. Mandal S, Van Treuren W, White RA, Eggesbo M, Knight R, Peddada SD. 2015. Analysis of composition of microbiomes: a novel method for studying microbial composition. Microb Ecol Heal Dis 26.

58. Morton JT, Marotz C, Washburne A, Silverman J, Zaramela LS, Edlund A, Zengler K, Knight R. 2019. Establishing microbial composition measurement standards with reference frames. Nat Commun $\mathbf{1 0}$.

59. Fernandes, D. A, Reid, J., Macklaim, M. J, McMurrough, T.A, Edgell, D.R., Gloor, B. G. 2014. Unifying the analysis of high-throughput sequencing datasets: characterizing RNA-seq, 16S rRNA gene sequencing and selective growth experiments by compositional data analysis. Microbiome 2:1-13.

60. Brill B, Amir A, Heller R. 2020. Testing for differential abundance in compositional counts data, with application to microbiome studies.

61. Jiang L, Amir A, Morton JT, Heller R, Arias-Castro E, Knight R. 2017. Discrete False-Discovery Rate Improves Identification of Differentially Abundant Microbes. mSystems 2:1-15. 